\title{
A GATA Transcription Factor from Soybean (Glycine max) Regulates Chlorophyll Biosynthesis and Suppresses Growth in the Transgenic Arabidopsis thaliana
}

\author{
Chanjuan Zhang ${ }^{1}$, Yi Huang ${ }^{1}$, Zhiyuan Xiao ${ }^{1}$, Hongli Yang ${ }^{1}$, Qingnan Hao ${ }^{1} \oplus$, Songli Yuan ${ }^{1}$, \\ Haifeng Chen ${ }^{1}$, Limiao Chen ${ }^{1}$, Shuilian Chen ${ }^{1}$, Xinan Zhou ${ }^{1, *}$ and Wenjun Huang ${ }^{2,3, *}$ \\ 1 Key Laboratory of Biology and Genetic Improvement of Oil Crops, Ministry of Agriculture and Rural Affairs, \\ Oil Crops Research Institute of Chinese Academy of Agricultural Sciences, Wuhan 430062, China; \\ zhangchanjuan@caas.cn (C.Z.); huangyi@oilcrops.cn (Y.H.); xiaozhiyuan@caas.cn (Z.X.); \\ yanghongli@caas.cn (H.Y.); haoqingnan@caas.cn (Q.H.); songliyuan@caas.cn (S.Y.); \\ chenhaifeng@caas.cn (H.C.); chenlimiao@caas.cn (L.C.); chenshuilian@caas.cn (S.C.) \\ 2 Key Laboratory of Plant Germplasm Enhancement and Specialty Agriculture, Wuhan Botanical Garden, \\ Chinese Academy of Sciences, Wuhan 430074, China \\ 3 Innovative Academy of Seed Design, Chinese Academy of Sciences, Beijing 100101, China \\ * Correspondence: zhouxinan@caas.cn (X.Z.); wjhuang@wbgcas.cn (W.H.); Tel.:+86-27-8671-1563 (X.Z.)
}

Received: 21 July 2020; Accepted: 11 August 2020; Published: 15 August 2020

\begin{abstract}
Chlorophyll plays an essential role in photosynthetic light harvesting and energy transduction in green tissues of higher plants and is closely related to photosynthesis and crop yield. Identification of transcription factors (TFs) involved in regulating chlorophyll biosynthesis is still limited in soybean (Glycine max), and the previously identified GmGATA58 is suggested to potentially modulate chlorophyll and nitrogen metabolisms, but its complete function is still unknown. In this study, subcellular localization assay showed that GmGATA58 was localized in the nucleus. Histochemical GUS assay and qPCR assay indicated that GmGATA58 was mainly expressed in leaves and responded to nitrogen, light and phytohormone treatments. Overexpression of GmGATA58 in the Arabidopsis thaliana ortholog AtGATA21 (gnc) mutant complemented the greening defect, while overexpression in Arabidopsis wild-type led to increasing chlorophyll content in leaves through up-regulating the expression levels of the large of chlorophyll biosynthetic pathway genes, but suppressing plant growth and yield, although the net photosynthetic rate was slightly improved. Dual-luciferase reporter assay also supported that GmGATA58 activated the transcription activities of three promoters of key chlorophyll biosynthetic genes of soybean in transformed protoplast of Arabidopsis. It is concluded that GmGATA58 played an important role in regulating chlorophyll biosynthesis, but suppressed plant growth and yield in transgenic Arabidopsis.
\end{abstract}

Keywords: Glycine max; soybean; GATA; transcription factor; chlorophyll; photosynthesis; growth; nitrogen

\section{Introduction}

Photosynthesis, a major contributor to crop yield, predominantly takes place in leaves where chlorophyll, one of the most abundant biological molecules in higher plants, plays unique and essential roles in photosynthetic light harvesting and energy transduction [1]. It has been reported that chlorophyll concentration, specific leaf weight or area and photosynthetic rate have impacts on crop yield, including soybean [2-4]. Among them, photosynthetic rate in leaves highly correlates 
with crop yield [5]. Thus, it is suggested that improving photosynthetic efficiency in soybean through biotechnology is a promising alternative way to increase crop yield [6]. Further, it was previously documented that the photosynthetic rate of soybean was highly correlated with chlorophyll content in leaves [7]. An increase in chlorophyll content provides an improved capacity to convert light energy to chemical energy and enhanced carbohydrate accumulation [8]. Therefore, it is possible to improve photosynthesis rate and crop yield through increasing chlorophyll content in leaves.

Chlorophyll consists of a chlorin ring and a geranylgeranyl diphosphate-derived isoprenoid, which are produced by tetrapyrrole and methylerythritol phosphate (MEP) biosynthetic pathways, respectively [9]. To date, the chlorophyll biosynthetic pathway has been clearly elucidated and almost all genes encoding enzymes involved in this pathway have been identified in Arabidopsis [1]. Moreover, multiple environmental stimulants and endogenous effectors are found to control the expressions of chlorophyll biosynthetic genes, but the transcription factors (TFs) involved in the regulation of chlorophyll biosynthetic pathway are little reported $[10,11]$. There are only several TFs found to date, primarily from Arabidopsis to regulate chlorophyll biosynthesis, including LONG HYPOCOTYL 5 (HY5), PHYTOCHROME-INTERACTING FACTORs (PIFs), GOLDEN2-LIKE (GLK) and GATA TFs $[10,11]$. Most of them are also involved in the light signaling pathway and photomorphogenesis, such as HY5 and PIFs that regulate chlorophyll biosynthesis through binding to G-box-containing promoter regions of certain key chlorophyll pathway genes [11]. Two renowned members of class $B$ GATA TFs (B-GATAs), GNC (GATA, NITRATE-INDUCIBLE, CARBON METABOLISM-INVOLVED) and its paralogous gene GNL/CGA1 (GNC-LIKE/CYTOKININ-RESPONSIVE GATA TRANSCRIPTION FACTOR1; referred to hereafter as GNL) have been identified in Arabidopsis to regulate chlorophyll biosynthesis and chloroplast formation [12-14].

GATA TFs are evolutionarily conserved proteins that contain a characteristic type IV zinc-finger DNA-binding domain and recognize a consensus sequence $(A / T) G A T A(A / G)$ of target promoter regions [15]. In plants, they play diverse roles in developmental control, responses to environmental stresses, hormones as well as nitrogen metabolism [16]. For example, a poplar GATA gene, PdGATA19/PdGNC was recently identified to not only regulate chlorophyll biosynthesis and also promote photosynthetic rate and plant growth [17]. Although the GATA gene family has been identified in Arabidopsis [15,18], rice [15], grape [19] and soybean [20], but only members have been functionally characterized to date. In soybean, the Glyma12g08131 encoding a GATA factor has been found to be possibly involved in nitrogen metabolism [21]. Nitrogen is essential to crop growth and yield, and nitrogen metabolism is closely related to chlorophyll metabolism and photosynthesis [22,23]. Moreover, nitrogen status in the leaves of several major crops, including soybean, can be rapidly, precisely and easily determined by a chlorophyll meter which reflects chlorophyll content [24,25]. Recently in our lab, two GATA members, GmGATA44 and GmGATA58 from a genome-wide analysis of GATA TFs family in soybean were found to be potentially involved in regulation of nitrogen and chlorophyll metabolisms [20]. GmGATA44 had been demonstrated to genetically complement the reduced chlorophyll phenotype of the Arabidopsis gnc mutant. It is a pair of paralogous genes GNC and GNL that contribute to chlorophyll biosynthesis and chloroplast formation in Arabidopsis seedlings [14]. However, the biological role of GmGATA58, a paralogous gene with GmGATA44, in chlorophyll biosynthesis, photosynthetic rate, growth and yield is still unknown. Therefore, in this study, the function of GmGATA58 TF was fully characterized to provide new insights into understanding the regulatory mechanism of chlorophyll biosynthesis and a potential tool gene for improving soybean growth and yield through biotechnology in future.

\section{Results}

\subsection{Sequence Analysis of GmGATA58}

The full-length cDNA of GmGATA58 gene was amplified from soybean variety 'TianLong No.1' and it contained an entire ORF (open reading frame) of $969 \mathrm{bp}$, encoding a protein of 322 amino acid 
residues with a predicted molecular weight of $36.2 \mathrm{kDa}$ and $\mathrm{pI}$ of 9.49. GmGATA58 contained the highly conserved GATA zinc finger domain and also a conserved leucine-leucine-methionine (LLM) domain closely located at the C-terminus as GmGATA44, AtGNC and AtGNL factors [26] (Figure S1). GmGATA58, identified previously as a member of B-GATA TF family [20], was further classified into the long B-GATA subfamily according to the structural criteria described by Behringer et al. [26] due to the long region of the N-terminal to the GATA domain. In addition, GmGATA58 showed a high similarity with GmGATA44 (88\% identity) at amino acid level, but only $44 \%$ and $41 \%$ identities with AtGNC and AtGNL protein, respectively. However, within the conserved GATA domain region, GmGATA58 shared $76 \%$ and $70 \%$ identities with AtGNC and AtGNL, respectively.

\subsection{GmGATA58 Localizes in Nucleus}

In eukaryotes, TFs are produced in cytoplasm but function in nucleus to regulate the transcription of downstream genes. TFs are generally transported from cytoplasm to nucleus via nuclear localization signal (NSL). Correspondingly, GmGATA58 as a TF was predicted bioinformatically to contain a putative NLS and thus localize in nucleus (Figure S1). In order to further confirm this prediction, the subcellular localization of GmGATA58 in leaves of Nicotiana benthamiana was analyzed using Agrobacterium-mediated transient expression. The results showed the GFP::GmGATA58 fusion protein driven by the CaMV $35 S$ promoter produced a strong green fluorescent signal only in the nucleus, which completely overlaid the red fluorescence produced from AtFib2-mCherry, a well-known nucleolar marker [27] (Figure 1). Meantime, the positive control GFP protein alone driven by the CaMV 35S promoter produced strong green fluorescent signals in whole cells (Figure 1). These results suggested that GmGATA58 was indeed localized in nucleus.

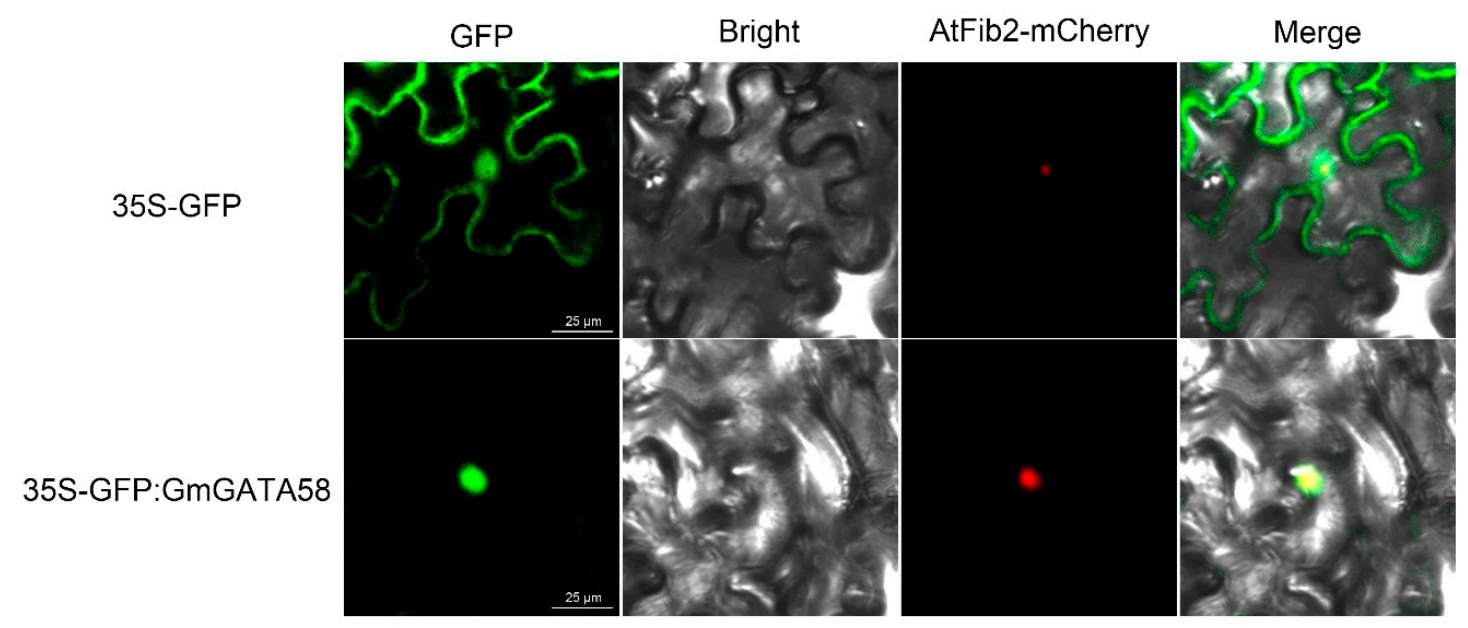

Figure 1. Subcellular localization of GmGATA58 protein in leaves of Nicotiana benthamiana using Agrobacterium-mediated transient expression. The plasmid construct containing GFP alone or GFP::GmGATA58 fusion protein was co-transformed into leaves of N. benthamiana with AtFib2-mCherry construct (nucleolar marker) and fluorescence signals were visualized using a confocal laser scanning microscopy $3 \mathrm{~d}$ after infiltration. GFP-(green), mCherry-(red), white-and their merge images were shown. Scale bar $=25 \mu \mathrm{m}$.

\subsection{Expression Pattern of GmGATA58}

Expression pattern of GmGATA58 in various tissues of soybean was firstly investigated by qPCR assay. The result indicated that GmGATA58 was predominately expressed in leaves, relatively moderately in immature and mature seed and very lowly in other tissues (Figure 2A). GmGATA58 and GmGATA44 were previously found to respond to nitrogen stress [20]. Here, GmGATA58 expression under the $7.5 \mathrm{mM}$ solution of normal nitrogen $(\mathrm{N}+)$ and the $0.75 \mathrm{mM}$ solution of low nitrogen $(\mathrm{N}-)$ conditions was also analyzed (Figure 2B). Although the expression level of GmGATA58 did not change 
considerably throughout the whole stages of either $\mathrm{N}+$ or $\mathrm{N}$ - treatment, but it was significantly higher under $\mathrm{N}+$ condition than that under $\mathrm{N}$ - condition at each sampling point, and their greatest difference between $\mathrm{N}+$ and $\mathrm{N}$ - treatments occurred at $216 \mathrm{~h}$ after treatment (Figure 2B). In other words, GmGATA58 was largely expressed under normal nitrogen condition and suppressed by low nitrogen stress. In addition, GmGATA58 expression under light and phytohormones treatments was also investigated as a number of cis-acting regulatory elements involved in light and phytohormones responses were predicted in its promoter sequence (Table S1). The results showed that GmGATA58 mRNA abundance considerably declined in leaves of soybean seedlings when seedlings removed out of a 3-day dark treatment were exposed to light treatment for 1 day and 3 days (Figure 2C). In response to 6-BA and 2, 4-D treatment, GmGATA58 transcript level decreased largely from 2 to $24 \mathrm{~h}$ after treatments (Figure 2D), but under $\mathrm{GA}_{3}$ treatment GmGATA58 expression level increased at 8 and $24 \mathrm{~h}$, particularly at $24 \mathrm{~h}$ with the maximum up-regulation (Figure 2D), compared to the water control. In short, GmGATA58 was highly expressed in leaves and responded to nitrogen level, light and phytohormone treatments.
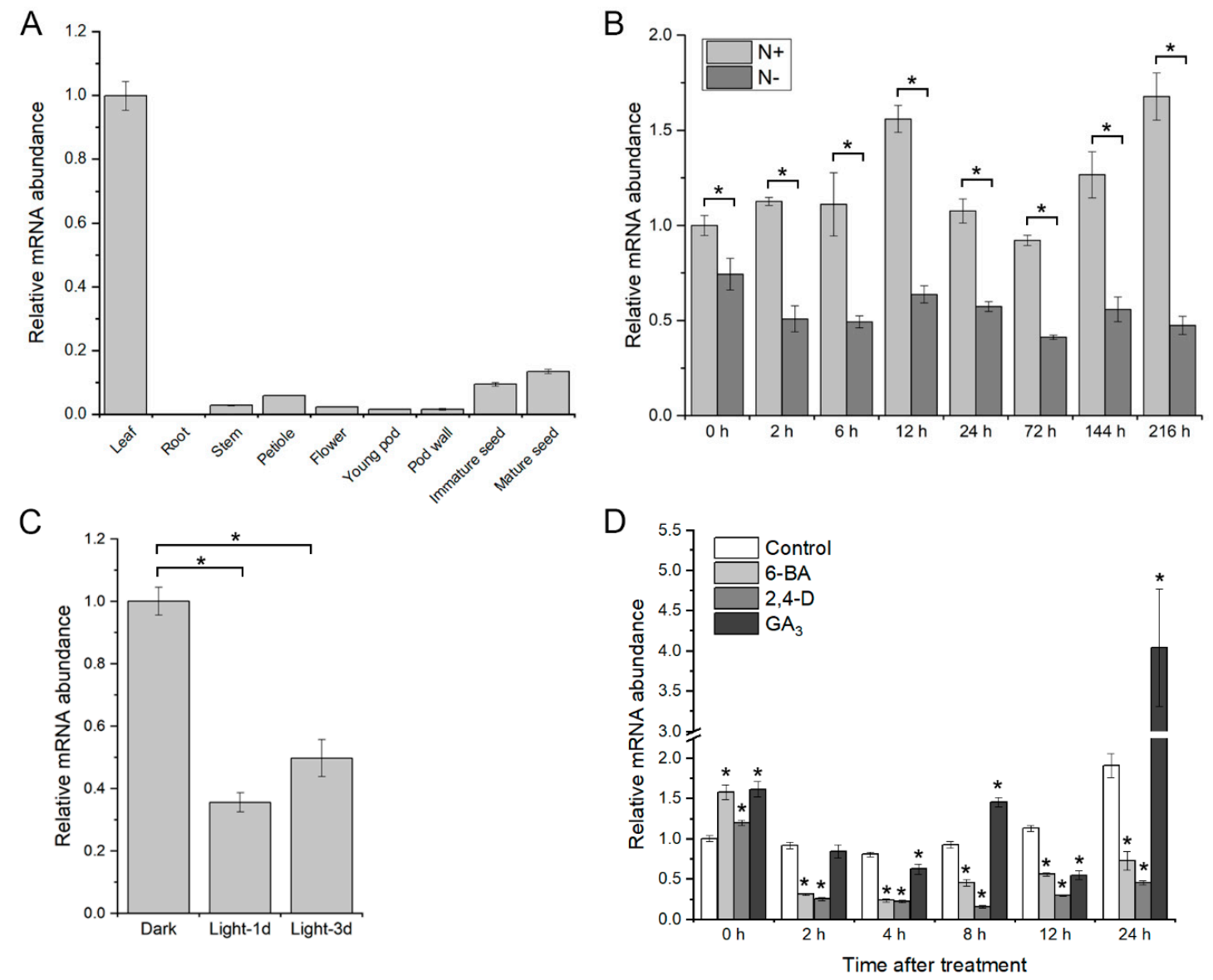

Figure 2. Expression pattern of GmGATA58 in various tissues of soybean (A) and in response to nitrogen (B), light/dark (C) and three phytohormones treatments (D). A, it is noted that pod wall sample are collected from immature pod. B, soybean seedlings were grown in the $7.5 \mathrm{mM}$ solution of normal nitrogen $(\mathrm{N}+)$ or the $0.75 \mathrm{mM}$ solution of low nitrogen $(\mathrm{N}-)$ conditions for up to 9 days. $\mathrm{C}$, soybean seedlings were grown in continuous dark condition for 3 days and then transferred to continuous light condition for up to 3 days. D, the 15 -day-old seedlings were sprayed with $100 \mu \mathrm{M}$ 6-BA, $100 \mu \mathrm{M}$ 2, 4-D or $100 \mu \mathrm{M} \mathrm{GA} 3$ phytohormones and distilled water as the control. The leaves of seedlings treated with nitrogen, light and phytohormones were harvested for GPCR assay at the different times. The GmACT11 gene was used as a reference gene and column values represented mean $\pm \mathrm{SD}$ (standard deviation). Asterisks $\left({ }^{*}\right)$ represent significant difference between groups or against the control in hormones treatment at the level of $p<0.05$. 


\subsection{Histochemical GUS Assay of Transgenic Arabidopsis Expressing GmGATA58 Promoter}

The preferable expression of GmGATA58 in soybean leaves was demonstrated by the qPCR results above. The histochemical GUS assay was also carried out to further analyze the spatial and temporal expression pattern of GmGATA58 in transgenic Arabidopsis expressing the GmGATA58 promoter. The results indicated that no GUS activities were detected in any tissue of wild-type plant as expected (Figure 3A-F), while in transgenic plants carrying the GmGATA58 promoter showed a different spatial and temporal expression pattern (Figure 3G-L). GmGATA58 was strongly expressed in cotyledons and young true leaves of both the 5- and 13-day-old seedlings, but weakly expressed in their root and rarely expressed in hypocotyls (Figure 3G,H). In transformed adult Arabidopsis plants, the expression of GmGATA58 was strongly detected in rosette leaves (Figure 3I), but lowly expressed in reproductive tissues, such as silique and flower except sepal with a moderate expression (Figure 3J-L). Overall, GmGATA58 was strongly expressed in leaves throughout all developmental stages of plants.

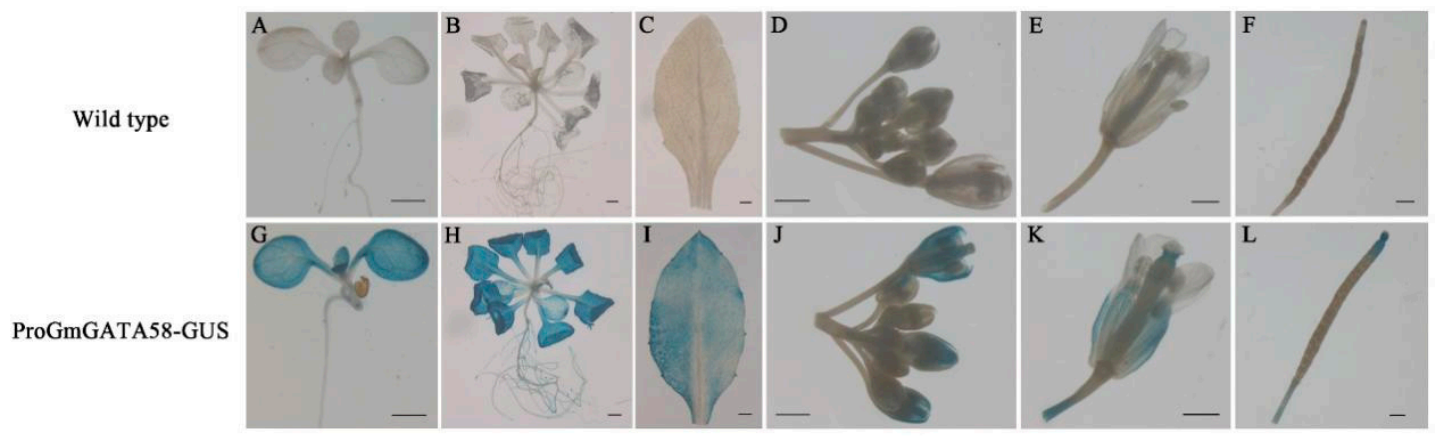

Figure 3. Histochemical GUS assay of transgenic Arabidopsis plants. The wild-type plant (A-F) and transgenic plant (G-L) carrying GmGATA58 promoter-GUS construct are indicated for GUS staining. Scale bars $=0.1 \mathrm{~cm}$.

\subsection{GmGATA58 Genetically Complements the gnc Mutant of Arabidopsis}

It was previously suggested that both GmGATA44 and GmGATA58 were likely to be involved in regulation of nitrogen and chlorophyll metabolisms in soybean, and the former had been demonstrated to regulate chlorophyll biosynthesis by genetic complementation of the Arabidopsis gnc mutant [20]. Therefore, overexpression of GmGATA58 under the control of CaMV 35S promoter in the gnc mutant background was also performed. Two independent $\mathrm{T}_{3}$ lines (gnc-OX7 and gnc-OX8) showing an obvious phenotypic change were selected for further analysis. The presence of introduced GmGATA58 in transgenic Arabidopsis plants was first confirmed by semi-quantitative RT-PCR assay (Figure 4A). GmGATA58 was strongly expressed in both gnc-OX7 and gnc-OX8 transgenic lines, and the endogenous AtGNC was expressed only in wild-type plant, but not in the gnc mutant and two transgenic lines (Figure 4A). Compared to the wild-type plants, the gnc mutant showed pale green leaves, but these pale green leaves were restored to normal green leaves of wild-type plants in both gnc-OX7 and gnc-OX8 transgenic lines irrespective of seedling or adult plants (Figure 4B and Figure S2). Moreover, the chlorophyll content in leaves also corresponded to the phenotypic complementation. The chlorophyll content in leaves was improved significantly in gnc-OX7 line compared to the gnc mutant, and even higher in gnc-OX7 line than wild-type plants but without significant difference (Figure 4C). These results demonstrated that GmGATA58 could genetically complement the gnc mutant of Arabidopsis as GmGATA44 did [20]. 


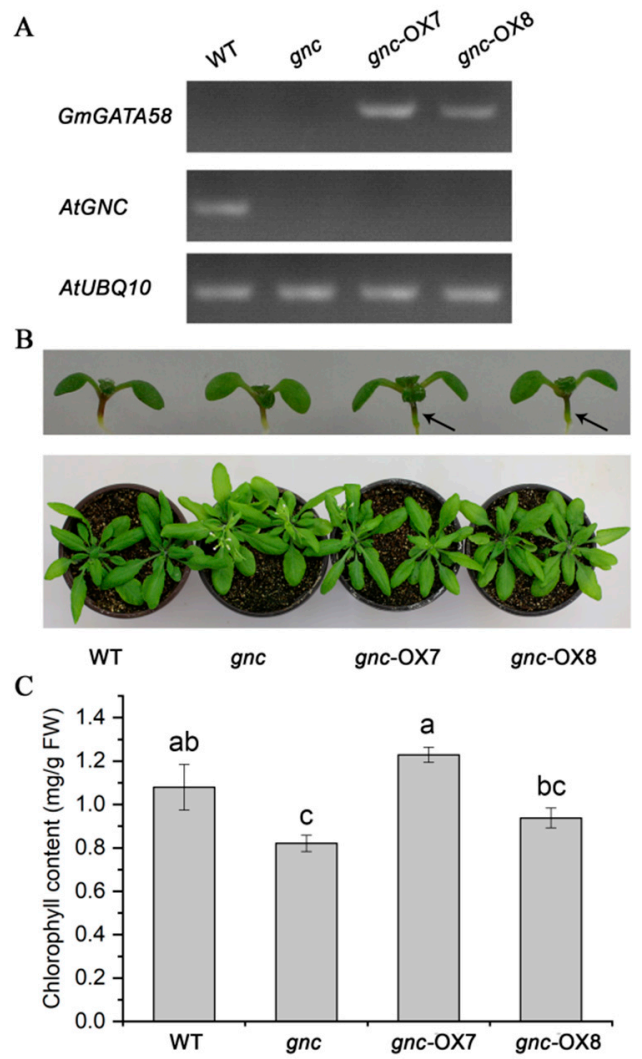

Figure 4. Genetical complementation of GmGATA58 gene in the Arabidopsis gnc mutant. (A) Semi-quantitative RT-PCR assay of GmGATA58 and AtGNC gene in the 3-week-old rosette leaves of wild-type (WT), gnc mutant, and two $\mathrm{T}_{3}$ transgenic lines overexpressing GmGATA58 in the gnc mutant background ( $g n c-\mathrm{OX} 7$ and gnc-OX8). The AtUBQ10 gene was used as an internal control. (B) Phenotypic comparison of wild-type, gnc mutant, and two transgenic lines at one week (upper panel) and four weeks (bottom panel) after germination. Arrows indicated dark green hypocotyls. (C) The chlorophyll content measurement in the rosette leaves of wild-type, gnc mutant and two transgenic lines at three weeks after germination. Data are presented as mean $\pm \operatorname{SD}(n=10)$ from three independent measurements. Means not sharing any lowercase letter are significantly different by the Tukey test at the $5 \%$ level of significance.

2.6. GmGATA58 Overexpression in Arabidopsis Increases Chlorophyll Content and Up-Regulates the Expression Levels of Chlorophyll Biosynthetic Genes

Considering the genetical complementation of GmGATA58 in the Arabidopsis gnc mutant background, overexpression of GmGATA58 in the wild-type Columbia of $A$. thaliana was also carried out. Two independent $\mathrm{T}_{3}$ overexpression lines (OX26 and OX40) with a high expression level of GmGATA58 and a clear phenotypic change were selected for further analysis. The green color of cotyledons and hypocotyls of the OX26 and OX40 transgenic seedlings was clearly strengthened, compared to the wild-type control (Figure 5A, upper panel). Moreover, this deepened green color in rosette leaves maintained throughout all development stages of the OX26 and OX40 lines (Figure 5A, middle and bottom panels). Similarly, the chlorophyll content in rosette leaves was highly correlated with the green color intensity. The OX26 and OX40 transgenic lines contained a higher level of chlorophyll in leaves than the wild type (Figure 5B). In addition, the plant growth and productivity were also altered by overexpression of GmGATA58, which were presented in detail later. Subsequently, the expressions of introduced GmGATA58 and chlorophyll biosynthetic pathway genes in Arabidopsis plants were also analyzed. The strong expression of GmGATA58 in leaves of the OX26 and OX40 transgenic lines was firstly confirmed by semi-quantitative PCR assay (Figure 5C), and then the large of chlorophyll biosynthetic genes were found to be up-regulated in either or both of the OX26 and OX40 
transgenic leaves by qPCR assay, including AtGSA1 (Glutamate-1-semialdehyde 2,1-aminotransferase), AtGSA2, AtCHLI1 (Magnesium chelatase I subunit), AtCHLI2, AtCHLD (Magnesium chelatase D subunit), AtCHLH (Magnesium chelatase $\mathrm{H}$ subunit), AtCHLM (Magnesium-protoporphyrin IX methyltransferase), AtPORA (Protochlorophyllide oxidoreductase), AtPORB, AtPORC, AtCHLP (Geranylgeranyl reductase) and AtCHLG (Chlorophyll synthase)(Figure 5D). Among them, the AtPORA gene which catalyzes a key step in tetrapyrrole biosynthetic pathway was most highly increased by 22-fold as well as AtPORB and AtPORC with relatively more expression levels (Figure 5D). These results indicated that GmGATA58 plays a certain role in modulating chlorophyll biosynthesis in Arabidopsis leaves through up-regulating the expression levels of a dozen of chlorophyll biosynthetic genes.
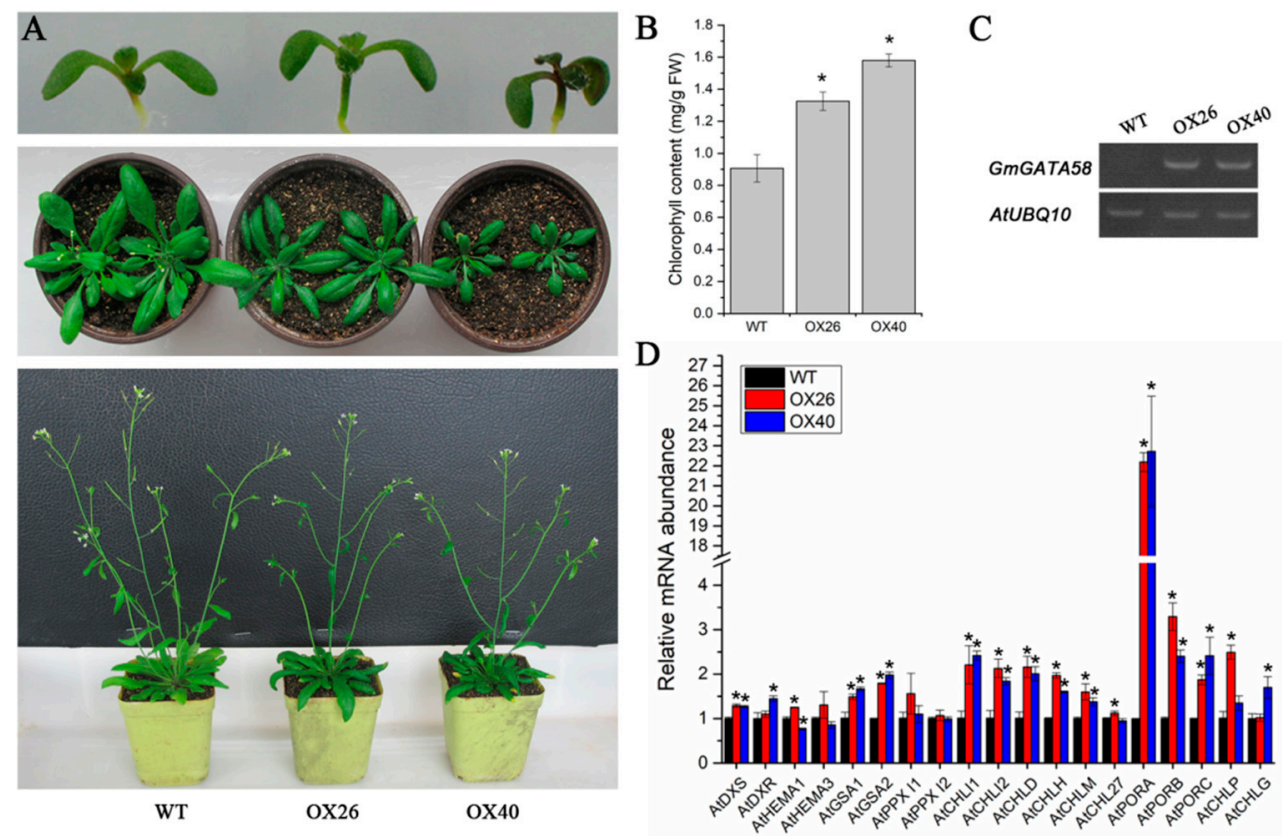

Figure 5. Overexpression analysis of GmGATA58 gene in Arabidopsis thaliana ecotype Columbia. (A) Phenotypic observation of the wild-type (WT) and two independent $\mathrm{T}_{3}$ overexpression lines (OX26 and OX40) at one week (upper panel), four weeks (middle panel) and five weeks (bottom panel) of post-germination. (B) The chlorophyll content measurement in three-week-old rosette leaves of WT, the OX26 and OX40 transgenic plants. Data are presented as mean \pm SD error bar $(n=10)$ from three independent measurements. Asterisks $\left(^{*}\right)$ indicate significant differences against the wild-type plant at $p<0.05$. (C) Semi-quantitative assay of GmGATA58 gene in rosette leaves of WT, the OX26 and OX40 transgenic plants. AtUBQ10 gene was used as the internal reference gene. (D) qPCR assay of chlorophyll biosynthetic pathway genes in the leaves of WT and two overexpression lines (OX26 and OX40). Data are normalized using AtGAPDH as the reference gene and shown as a percentage of the expression in the wild-type plant. Asterisks $\left(^{*}\right)$ indicate significant differences against the wild-type plant at $p<0.05$.

\subsection{Plant Growth Vigor and Productivity of Arabidopsis Are Suppressed by Overexpression of GmGATA58}

In addition to the change of chlorophyll content and leaf color mentioned above, the growth vigor and productivity of Arabidopsis plants were also altered by GmGATA58 overexpression. Corresponding to the increase of chlorophyll content in leaves, the net photosynthetic rate was also improved slightly by ectopic expression of GmGATA58 (Figure 6A), but the plant growth was suppressed. Compared to the wild-type plant, the two overexpression lines OX26 and OX40 revealed smaller leaves and a shorter inflorescence axis (Figures 5A and 6B), and their productivities per plant including seed weight, dry mass of aerial part and their ratios (defined as harvest index) were also decreased, especially for OX40 lines (Figure 6C). Additionally, the flowering time of the overexpression lines was also significantly 
delayed (Figure 5A, second panel). These results suggested that ectopic expression of GmGATA58 in Arabidopsis had a negative effect on plant growth and productivity.
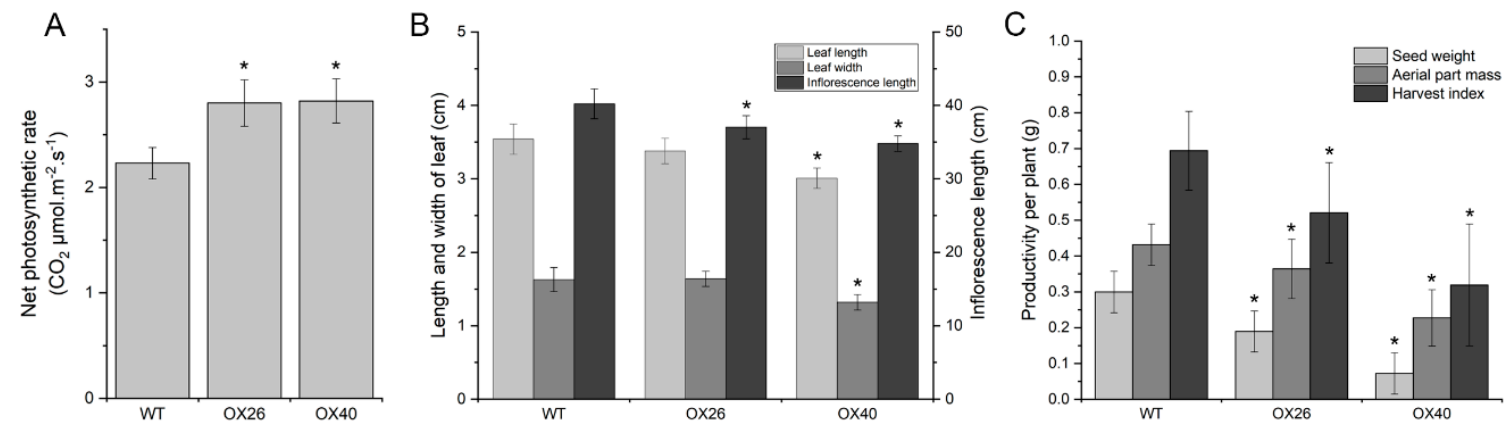

Figure 6. Growth and productivity alteration of the wild-type plants and two GmGATA58 overexpression transgenic lines of Arabidopsis thaliana. The net photosynthetic rate (A), growth vigor (B) and productivity per plant (C) were measured in the wild-type (WT) plant and two overexpression lines OX26 and OX40. Data are presented as mean \pm SD bar, and asterisks $\left(^{*}\right)$ indicate significant differences against the WT plant at the level of $p<0.05$.

\subsection{Transcription Activation of GmGATA58 against the Promoters of Chlorophyll Biosynthetic Genes from} Soybean in Transiently Transformed Arabidopsis Protoplast

A dozen of chlorophyll biosynthetic pathway genes were up-regulated by ectopic expression of GmGATA58 in Arabidopsis plants, which suggests that GmGATA58 is able to bind to their promoters and activate their transcription expression. Similarly, whether GmGATA58 has an ability to interact with and activate the promoters of key chlorophyll biosynthetic genes in soybean (GmCHLI1, GmCHLH1 and GmCHLH3) was also investigated in transiently transformed Arabidopsis protoplast using a dual-luciferase reporter assay. The results indicated that the protoplasts co-transformed with GmGATA58 and anyone of three soybean promoters produced a 1.5- to 5.4-fold higher value of LUC/REN fluorescence activity than the control protoplasts only transformed with the soybean promoters without $G m G A T A 58 \mathrm{TF}$ and, among them, the transcriptional activity of $G m C H L H 3$ promoter was increased to the highest level (6.4-fold) by GmGATA58 activation (Figure 7). The result suggests that GmGATA58 can also bind to these three promoters of chlorophyll biosynthetic genes from soybean and activate their transcription in Arabidopsis protoplasts.
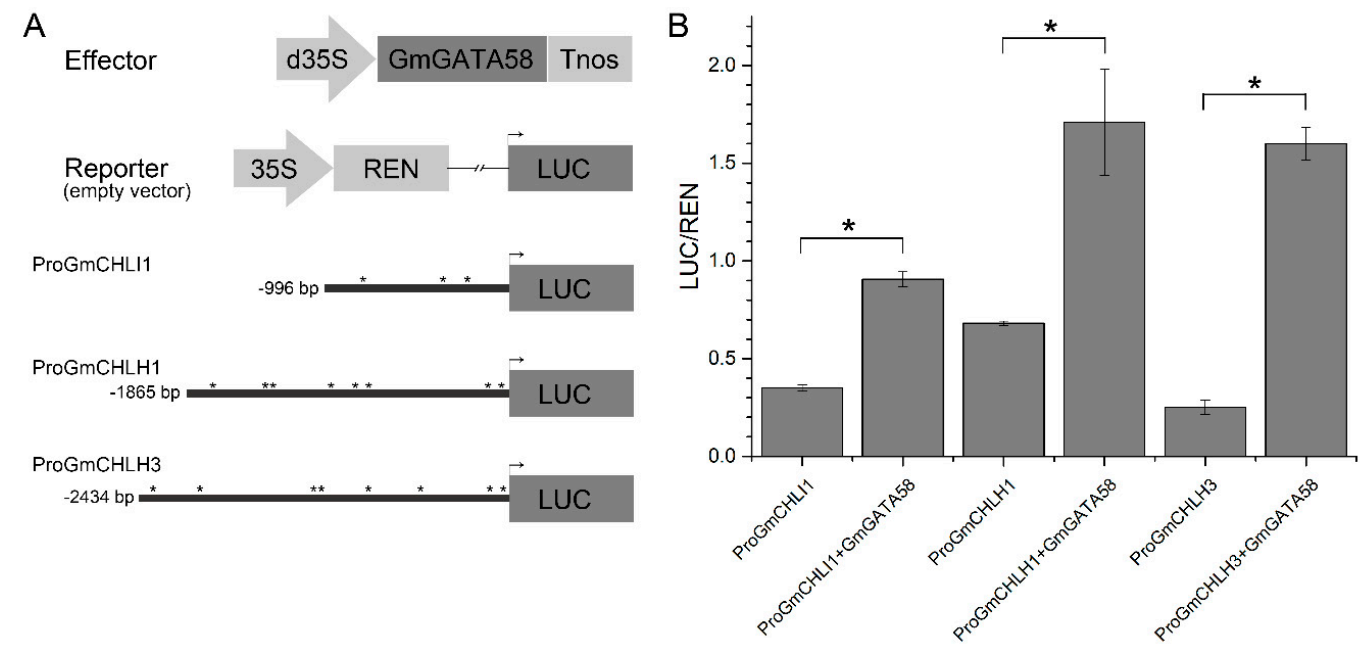

Figure 7. Dual-luciferase reporter assays of GmGATA58 and three promoters of chlorophyll biosynthetic genes (GmCHLI1, GmCHLH1 and GmCHLH3) from soybean in transiently transformed protoplast of Arabidopsis. (A) Schematics of effector and reporter constructs for dual-luciferase reporter assay. d35S, 
double CaMV 35 S promoter; Tnos, NOS terminator; REN, Renilla luciferase; LUC, Firefly luciferase; Asterisks $\left({ }^{*}\right)$ mean GATA binding site $(\mathrm{A} / \mathrm{T}) \mathrm{GATA}(\mathrm{A} / \mathrm{G})$ in the promoter sequences, which were predicted by New PLACE tool online. Numbers indicated the length of promoter sequence upstream of the translation start codon. (B) Dual luciferase reporter assays of GmGATA58 against GmCHLI1, GmCHLH1 and GmCHLH3 promoters. Relative luciferase activity was determined by the ratio of LUC/REN activity. Each column represents mean \pm SD error bar and asterisks $\left(^{*}\right)$ represent $p<0.05$.

\section{Discussion}

GATA factors are considerably conserved within the GATA zinc finger domain, but not highly conserved at overall protein level. A low similarity of protein sequence of GmGATA58 with AtGNC and AtGNL was found (Figure S1), which is consistent with the sequence identity between AtGNC and AtGNL. However, GmGATA58 and GmGATA44, as well as AtGNC and AtGNL had a close phylogenetic relationship and formed a distinct cluster when the conserved GATA domains were used for phylogenetic tree construction [20]. It is well documented that both GNC and GNL play key roles in chloroplast development, modulating chlorophyll biosynthesis, chloroplast number, size and total leaf starch [12,13]. Therefore, the similar function of GmGATA58 in transgenic Arabidopsis was also confirmed. GmGATA58 not only played an important role in modulating chlorophyll biosynthesis, but also unexpectedly showed impacts on growth vigor, flowering time and agricultural productivity in transgenic Arabidopsis.

The reduced chlorophyll content in leaves and thus greening defect have been previously regarded as the typical phenotypes of the $g n c$ and $g n l$ mutants of Arabidopsis $[18,28,29]$, while the GNC and GNL overexpressing Arabidopsis seedlings produced dark green leaves and accumulated more chlorophyll $[12,13,26]$. Here, our results also indicated that overexpression of GmGATA58 in the gnc mutant was able to complement the greening defect (Figure 4), while overexpression in Arabidopsis wild-type plants caused an increased chlorophyll content and dark green leaves (Figure 5). The recently identified poplar $P d G N C$ also revealed a similar result when overexpressed in poplar [17]. These results suggested that GmGATA58 shared a conserved function in modulating chlorophyll biosynthesis with GNC and GNL from Arabidopsis and poplar. Correspondingly, the expression levels of the large of chlorophyll biosynthetic pathway genes in Arabidopsis overexpression lines were improved, especially AtPORA/B/C, AtCHLI1/2, AtCHLD and AtCHLH (Figure 5D). Several similar studies have also been reported previously that the expression level of certain key genes of chlorophyll biosynthesis highly correlated with the chlorophyll content of leaves in the gnc and/or gnl mutants or their overexpression transgenic plants $[14,28,30]$. They found that several key chlorophyll biosynthetic genes, including AtHEMA3 (Glutamyl tRNA reductase), AtGSA1/2, AtCHLI1/2, AtCLHM and AtPORBA/B/C were regulated by GNC and GNC expressions [14,17]. However, AtHEMA genes, considered as a rate-limited gene for tetrapyrrole synthesis were not improved by ectopic expression of GmGATA58 (Figure 5D). In addition, whether GmGATA58 is able to bind to the promoters of chlorophyll pathway genes from soybean and activate their expression was also investigated in transformed Arabidopsis protoplast using a dual-luciferase reporter assay (Figure 7). All the three selected promoters from GmCHLI1, GmCHLH1 and $\mathrm{GmCHLH3}$ were activated by GmGATA58. These soybean genes overlapped with the Arabidopsis chlorophyll genes which were up-regulated by ectopic expression of GmGATA58 (Figure 5D). It is noted that soybean PORA gene corresponding to AtPORA gene with highest up-regulation was not included in dual-luciferase reporter assay, as our transcriptome results from GmGATA44 overexpressing soybean transformants indicated that GmPORA, as well as GmPORB and GmPORC were not significantly changed (data not shown), but their relationships with GmGATA58 should be further analyzed in next step.

In addition to regulating chlorophyll biosynthesis and chloroplast development, both GNC and GNL can also control a number of developmental events, including germination, expansion growth, flowering time and senescence $[12,16,28,31]$. Similarly, GmGATA58 showed a wide range of functions besides regulating chlorophyll synthesis and accumulation. Overexpression of GmGATA58 in the 
wild-type Arabidopsis led to delayed seed germination and flowering time as well as reduced rosette leaf size. Specifically, seeds from the transgenic lines, especially OX40 line, germinated 1-2 d slower than wild-type seeds (data not shown), and flowering time of OX26 and OX40 lines was obviously delayed compared to that of the wild type. These phenotypic changes are consistent with the previous reports that seeds germinated faster, rosette leaf size enlarged and plants flowered earlier for the gnc and $g n l$ mutants [28], whereas the GNC and GNL overexpressing plants showed a slower seed germination and flowering time, compared to the wild-type plants [26,32]. In addition, overexpression of other LLM domain-containing B-GATA factors, including SlGATA4, SlGATA5 and SlGATA7 from tomato (Solanum lycopersicon) as well as BdGATA4 and BdGATA6 from Brachypodium (Brachypodium distachyon) in Arabidopsis, also produced a similar phenotype [26]. They hypothesized that the LLM domain-containing B-class GATA TFs were functionally conserved among different species. Our results presented here also strongly supported this hypothesis.

It is interesting and unexpected that the net photosynthetic rate in leaves was slightly improved, but the plant growth and agricultural productivity per plant were clearly reduced in the GmGATA58 overexpression lines (Figures 5 and 6), which was strongly contrary to the report of PdGNC [17]. Overexpression of PdGNC in poplar led to faster growth, higher biomass accumulation and an increase in chlorophyll content, photosynthetic rate and plant height. In this study, the transgenic plants overexpressing GmGATA58 grew weakly at both young and adult stages with smaller leaves and shorter inflorescences, compared to the wild-type control, which possibly caused a low seed yield and biomass of aerial part per plant (Figure 6). In fact, a lot of the evidence showed that there is no correlation between the yield of a wide range of crops and photosynthesis, and the yield is limited by sinks for photosynthates, but not by photosynthetic capacity [4]. The improvement of photosynthetic efficiency also contributes to a greater yield. Although the net photosynthetic rate is slightly improved in leaves by overexpression of GmGATA58, photosynthetic energy conversion efficiency and photosynthate transportation efficiency may not be up-regulated. Moreover, it is suggested that GATA genes share both conserved and diverged functions between herbaceous and woody plants as PdGNC showed a similar and a different roles with AtGNC and AtGNL [17]. Perhaps, that is why GmGATA58-expressing transformants revealed some contrary phenotypic traits with the PdGNC-expressing transformants.

In plants, photosynthesis takes place primarily in chloroplasts of leaves, which contain chlorophyll. Since GmGATA58 played an important role in modulating chlorophyll biosynthesis, it should be highly expressed in leaf tissue. Our qPCR assay and GUS staining assay results also supported this assumption (Figures 2A and 3). As previously demonstrated, GNC and GNL paralogs are induced by nitrogen sources, cytokinin and light, but suppressed by GA and darkness $[13,18,28,33]$. However, the expression pattern of GmGATA58 in response to these factors showed partial difference, although the similar lightand phytohormone-responsive elements were found in the GmGATA58 promoter sequence (Table S1). The transcript level of GmGATA58 was also induced by high nitrogen condition and suppressed by low nitrogen status (Figure 2B). Nitrogen conditions have a marked effect on chlorophyll synthesis and they are highly correlated with each other [34]. GmGATA58 was remarkably down-regulated by 2, 4-D (Figure 2D), which agreed with the report on GNC and GNL [18,35]. By contrast, GmGATA58 expression was suppressed by light and 6-BA (Figure 2C,D) and promoted by GA 3 treatment (Figure 2D), which were partially contrary to the previous report for GNC and GNL. Light is an important external signal and plant hormones as internal signals coordinate control plant growth and development, including chlorophyll biosynthesis [36]. The de-etiolation process takes place when dark-grown seedlings are exposed to light, accompanied with the accumulation of chlorophyll, and multiple endogenous phytohormones are also involved. Previous studies showed that AtGNC/GNL positively responded to light and cytokinin, and negatively to auxin and gibberellins $[28,33,35]$. In this study, the reasonable explanation for the suppression of GmGATA58 by light and the promotion by $\mathrm{GA}_{3}$ is still unknown, and there may be other regulatory proteins affecting GmGATA58 expression via interaction or coordination. It is also possible that GmGATA58 is functionally redundant in regulating chlorophyll biosynthesis as its paralogous GmGATA44 also played an important role in chlorophyll 
biosynthesis [20]. In summary, GmGATA58 was induced by nitrogen and responded to light and hormone treatments, but the responses did not exactly coincide with Arabidopsis GNC and GNL.

\section{Materials and Methods}

\subsection{Plant Materials}

Plants of soybean (Glycine max) cultivar 'TianLong No.1' were grown under white light $\left(200 \mu \mathrm{mol} \cdot \mathrm{m}^{-2} \cdot \mathrm{s}^{-1}\right)$ and about $60 \%$ relative humidity with a $16 \mathrm{~h} \mathrm{light} / 8 \mathrm{~h}$ dark cycle at $28{ }^{\circ} \mathrm{C}$ in a plant growth chamber and various tissues including root, stem, leaf, petiole, flower, young pod, immature seed, pod wall of immature seed and mature seed were collected for qPCR assay. Soybean seedling in response to nitrogen treatment was carried out following the method by Zhang et al. [20] with minor modification. In brief, the 5-day-old soybean seedlings were planted in the mixture of vermiculite:perlite $=2: 1$ for $4 \mathrm{~d}$ and then the cotyledons of seedlings were cut off prior to nitrogen treatment. The seedlings were irrigated with half Hoagland solution with $7.5 \mathrm{mM}$ nitrogen as normal nitrogen condition for $4 \mathrm{~d}$, and then transferred to 10 times-diluted half Hoagland solution with $0.75 \mathrm{mM}$ nitrogen as low nitrogen stress $(\mathrm{N}-)$ or continued to be kept in the normal nitrogen condition $(\mathrm{N}+)$. The nutritional solution was refreshed every 4 days and the first fully expanded leaves were harvested for qPCR assay at different time points after treatment. For light treatment, the 15-day-old soybean seedlings were grown in the continuous dark for $3 \mathrm{~d}$ and then transferred to continuous light condition (about $100 \mu \mathrm{mol} \cdot \mathrm{m}^{-2} \cdot \mathrm{s}^{-1}$ ) for up to $3 \mathrm{~d}$. The first fully expanded leaves were harvested for qPCR assay. As for phytohormones treatments, the 15-day-old soybean seedlings grown in compost plastic pots in a naturally illuminated glasshouse were sprayed with $100 \mu \mathrm{M} 6-\mathrm{BA}, 100 \mu \mathrm{M} 2$, 4-D or $100 \mu \mathrm{M} \mathrm{GA}_{3}$ hormones. The seedlings sprayed with distilled water were used as control. The first fully expanded leaves from the apical bud of soybean were collected at $0,2,4,8,12$ and $24 \mathrm{ht}$ after treatment.

The wild-type Columbia and gnc mutant of A. thaliana were used for plant transformation. They were grown under white light $\left(100 \mu \mathrm{mol} \cdot \mathrm{m}^{-2} \cdot \mathrm{s}^{-1}\right)$ and $60 \%$ relative humidity with a $16 \mathrm{~h}$ light $/ 8 \mathrm{~h}$ dark cycle at $24{ }^{\circ} \mathrm{C}$. The gnc mutant contains a T-DNA insertion in the second exon of AtGATA21 (At5g56860) and produces greening defect with reduced chlorophyll content in pale green leaves. The gnc mutant line was obtained from the Arabidopsis Biological Resource Center (Stock No: SALK_001778). Arabidopsis plants were grown in a plant growth chamber at $24{ }^{\circ} \mathrm{C}$ under the condition of $16 \mathrm{~h}$ of light and $8 \mathrm{~h}$ of dark.

\subsection{DNA and RNA Extraction}

Genomic DNA from young leaves of soybean and $A$. thaliana was isolated using CTAB method [37]. Total RNA was extracted from various tissues of soybean and young leaves of transgenic Arabidopsis plants using Trizol reagent (Invitrogen, Carlsbad, CA, USA) according to the manufacturer's instruction. The quality and quantity of nucleic acids were evaluated by agarose gel electrophoresis and Epoch microplate spectrophotometer (BioTek, Winooski, VT, USA).

\subsection{Isolation of GmGATA58 gene}

Based on the sequence of Glyma.17G055200 (Phytozome, v12.0), the full-length cDNA clone of GmGATA58 was isolated from leaf cDNA template of soybean variety 'TianLong No.1' using the gene-specific primers (Table S2). The PCR product was cloned into the pMD18-T vector (Takara, Dalian, China and sequenced for further confirmation).

\subsection{Subcellular Localization of GmGATA58}

The coding region of GmGATA58 was amplified from pMD18-GmGATA58 recombinant plasmid using the gene-specific primers (Table S2) and then subcloned into the pEGAD binary vector with Sma I and BamH I double digestion to generate the CaMV 35S-GFP::GmGATA58 construct for subcellular localization assay. In this construct, GmGATA58 protein was fused to the C-terminus of GFP protein 
under the control of the CaMV $35 \mathrm{~S}$ promoter. This construct was introduced into Agrobacterium tumefaciens strain EHA105 by the freeze-thaw method. Agro-infiltration transient expression in leaves of Nicotiana benthamiana was used for subcellular localization assay of GmGATA58. The plasmid construct coding AtFib2-mCherry fusion protein was used as a nucleolar marker [27]. Leaves of N. benthamiana were infiltrated with Agrobacterium cultures to transiently express GFP protein alone or GFP::GmGATA58 fusion protein in combination with the AtFib2-mCherry nucleolar marker. After $3 \mathrm{~d}$ of infiltration, GFP (green) and mCherry (red) fluorescence signals were observed using a confocal laser scanning microscopy (Leica, Wetzlar, Germany).

\subsection{Isolation and Analysis of GmGATA58 Promoter}

A promoter region upstream of the start codon ATG (2013 bp) of GmGATA58 was amplified from soybean genomic DNA using the promoter-specific primers (Table S2) and subcloned into the pCXGUS-P vector [38] to generate the pCXGUS-GmGATA58p construct, in which the reporter GUS gene was expressed under the control of the GmGATA58 promoter. This construct was then introduced into Agrobacterium tumefaciens strain GV3101 by freeze-thaw method and transformed into A. thaliana Columbia wild-type plants using floral dip method [39]. For GUS histochemical staining, various tissues from wild-type and transgenic plants carrying the GmGATA58 promoter were harvested, treated with $90 \%$ acetone for $20 \mathrm{~min}$, rinsed with distilled water and incubated with GUS staining solution at $37^{\circ} \mathrm{C}$ overnight in dark. Finally, these tissues were destained using $70 \%$ ethanol prior to photographing under a microscope. In addition, the putative cis-acting regulatory elements in the promoter sequence of GmGATA58 were predicted using PlantCARE database [40].

\subsection{Overexpression Vector Construction and Arabidopsis Transformation}

The complete coding region of GmGATA58 was amplified from pMD18-GmGATA58 recombinant plasmid using the gene-specific primers (Table S2) and inserted into the intermediate vector pGWC, which can be digested with Ahd I enzyme to generate a T-vector. Subsequently, GmGATA58 was transferred from $\mathrm{pGWC}$ vector to $\mathrm{pB} 2 \mathrm{GW7}$ vector by LR reaction (Invitrogen, Carlsbad, CA, USA) to generate a pB2GW7-GmGATA58 overexpression construct, in which GmGATA58 expression was driven by the CaMV $35 \mathrm{~S}$ promoter. This overexpression construct was introduced into Agrobacterium tumefaciens strain GV3101 by freeze-thaw method. The wild-type Columbia plants and the homozygous mutant gnc of $A$. thaliana were used for genetic transformation via floral dip method [39].

Basta-resistant plants were further confirmed by PCR using the CaMV $35 S$ promoter specific primer with the GmGATA58 reverse primer. The Basta resistant screening was used in genetic segregation analysis to select homozygous lines. The expression level of GmGATA58 in transgenic lines was analyzed by semi-quantitative RT-PCR assay. Finally, a total of four independent homozygous $\mathrm{T}_{3}$ lines with a high expression level of GmGATA58 were selected for further study, including two GmGATA58 genetical complement lines in the gnc mutant background (gnc-OX7 and gnc-OX8) and two GmGATA58 overexpression lines in wild-type background (OX26 and OX40).

\subsection{Semi-Quantitative RT-PCR and Quantitative PCR Analysis}

After treated with RNase-free DNase I (Thermo Fisher Scientific, Waltham, MA, USA), one microgram of total RNA was used to synthesize fist-strand cDNA using M-MLV reverse transcriptase (Promega, Madison, WI, USA) according to the supplier's manual. The expression levels of AtGNC and GmGATA58 in leaves of Arabidopsis lines were analyzed by semi-quantitative RT-PCR. PCR amplification was performed using the following program: one denaturation cycle at $94{ }^{\circ} \mathrm{C}$ for $5 \mathrm{~min}$, 31 cycles of $94{ }^{\circ} \mathrm{C}$ for $30 \mathrm{~s}, 56{ }^{\circ} \mathrm{C}$ for $30 \mathrm{~s}$, and $72{ }^{\circ} \mathrm{C}$ for $30 \mathrm{~s}$, and a final extension at $72{ }^{\circ} \mathrm{C}$ for $10 \mathrm{~min}$. The amplified products were analyzed by electrophoresis on $1.0 \%$ agarose gel.

A qPCR assay was used to analyze the expression pattern of GmGATA58 in various tissues of soybean and soybean leaves under nitrogen, phytohormones and light treatments, and also the expression levels of chlorophyll biosynthetic genes in transgenic overexpression lines of Arabidopsis. 
The qPCR assay was carried out using SYBR Premix Ex Taq II kit (Takara, Dalian, China, following the manual's recommendation, and run on ABI7500 Fast Real-Time PCR equipment (Applied Biosystems, Foster, CA, USA). In brief, the reaction mixture contained $10 \mu \mathrm{L}$ of $2 \times$ SYBR Premix Ex Taq mix, $0.3 \mu \mathrm{M}$ each of forward and reverse primers, and $2 \mu \mathrm{L}$ of 5 -fold-diluted first-strand cDNA template. The qPCR program was as follows: $95^{\circ} \mathrm{C}$ for $30 \mathrm{~s}, 40$ cycles of $95^{\circ} \mathrm{C}$ for $3 \mathrm{~s}$ and $60{ }^{\circ} \mathrm{C}$ for $30 \mathrm{~s}$, and a default melting curve program. Three biological replicates were performed for each sample. Soybean ACT11 (Glyma.18G290800) gene and Arabidopsis GAPDH (At3g26650) gene were used as the internal controls for qPCR assay in soybean and Arabidopsis, respectively. The relative expression level of gene was analyzed using $2^{-\mathrm{ddCt}}$ method [41]. All primers were designed using Primer 5.0 software and listed in the Supplementary Table S2.

\subsection{Determination of Chlorophyll Content, Photosynthetic Rate and Growth in Arabidopsis}

Determination of chlorophyll content and photosynthetic rate in rosette leaves of Arabidopsis was carried out after three weeks of seed germination. The chlorophyll content was measured using a spectrophotometric method as previously described [42]. The net photosynthetic rate was measured using a LI-6400-XT portable photosynthesis system with a plant Arabidopsis chamber (Li-Cor, Lincoln, NE, USA) following the method by Xin et al. [43]. For measurement of chlorophyll content and net photosynthetic rate, a total of ten independent samples for each line were measured, and each sample was repeated in triplicate. In addition, the productivity per plant for each line, including seed weight, dried weight of aerial part of plant and their ratios (referred to harvest index) was determined from 25 to 30 independent individual plants.

\subsection{Dual-Luciferase Assay in Transiently Transformed Protoplast of Arabidopsis}

The promoter regions of GmCHLH1 (Glyma.03G137000) in length of $1865 \mathrm{bp}$, GmCHLH3 (Glyma.19G139300) in length of $2434 \mathrm{bp}$ and GmCHLI1 (Glyma.13G232500) in length of $996 \mathrm{bp}$ upstream of the start codon ATG were amplified from soybean genomic DNA using the promoter-specific primers (Table S2) and cloned into pGreen II 0800-LUC vector to generate ProGmCHLH1::LUC, ProGmCHLH3::LUC and ProGmCHLI1::LUC reporter plasmids. pGreenII0800-LUC vector without promoter insertion was used as a control. The effector plasmid was constructed by cloning the full-length coding region of GmGATA58 into the pAN580 vector under the control of the double 35S promoter. As described previously [44], the reporter plasmid was co-transformed with the effector plasmid or transformed alone into Arabidopsis protoplasts. The Pro35S:REN gene (Renilla luciferase) in the pGreenII-LUC vector was used as an internal control. The Dual-Luciferase Reporter assay system (Promega, Madison, WI, USA) was used to determine the ratio of firefly luciferase to Renilla luciferase (LUC/REN) to indicate the promoter activities.

\subsection{Statistical Analysis}

Data were presented as mean \pm SD (standard deviation) bar. Statistical separation of treatment means was by analysis of variance (ANOVA) using Originpro 2020b (OriginLab Corporation, Northampton, MA, USA) at the level of $p<0.05$. Graphs were also created using Originpro 2020b (OriginLab Corporation, Northampton, MA, USA).

\section{Conclusions}

As an LLM domain-containing B-class GATA TF, GmGATA58 was demonstrated to be involved in regulating chlorophyll biosynthesis in Arabidopsis as overexpression of GmGATA58 not only genetically complemented the greening defect of the gnc mutant, but also improved chlorophyll content in transgenic leaves through up-regulation of the expression levels of most of chlorophyll biosynthetic genes. Meanwhile, the net photosynthetic rate in transgenic leaves was also slightly improved, but unexpectedly the Arabidopsis growth and productivity were clearly suppressed. 
In addition, GmGATA58 was predominantly expressed in leaves and responded to nitrogen, light and phytohormone treatments.

Supplementary Materials: The following are available online at http://www.mdpi.com/2223-7747/9/8/1036/s1. Figure S1: Multiple alignment of deduced amino acids of GmGATA58 with other three GATA factors. Figure S2: Restoration of greening defect of Arabidopsis thaliana gnc mutant by GmGATA58 overexpression. Table S1: Putative cis-acting regulatory elements predicted in the GmGATA58 promoter sequence. Table S2: Primers used in this study for cloning, vector construction, semi-quantitative RT-PCR and qPCR assay.

Author Contributions: Conceptualization, C.Z., W.H. and X.Z.; investigation, C.Z., Z.X., Y.H. and H.Y.; data curation, Z.X., H.Y. and Q.H.; formal analysis, Q.H., H.C. and L.C.; validation and visualization, Y.H. and S.Y.; resources, S.C.; funding acquisition, X.Z.; wrote the paper, C.Z. and W.H. All authors have read and agreed to the published version of the manuscript.

Funding: This study was supported by grants from the National Transgenic Project (Grant No. 2016ZX08004-005) and the National Key R\&D Program of China (Grant No. 2017YFD0101500).

Acknowledgments: We want to thank everyone in our soybean team for their suggestions and help.

Conflicts of Interest: The authors declare no conflict of interest.

\section{References}

1. Eckhardt, U.; Grimm, B.; Hortensteiner, S. Recent advances in chlorophyll biosynthesis and breakdown in higher plants. Plant Mol. Biol. 2004, 56, 1-14. [CrossRef] [PubMed]

2. Buttery, B.R.; Buzzell, R.I.; Findlay, W.I. Relationships among photosynthetic rate, bean yield and other characters in field-grown cultivars of soybean. Can. J. Plant Sci. 1981, 61, 190-197. [CrossRef]

3. Maekawa, T.; Kokubun, M. Correlation of leaf nitrogen, chlorophyll and rubisco contents with photosynthesis in a supernodulating soybean genotype Sakukei 4. Plant Prod. Sci. 2005, 8, 419-426. [CrossRef]

4. Zhu, X.-G.; Long, S.P.; Ort, D.R. Improving photosynthetic efficiency for greater yield. Annu. Rev. Plant Biol. 2010, 61, 235-261. [CrossRef] [PubMed]

5. Zelitch, I. The close relationship between net photosynthesis and crop yield. BioScience 1982, 32, 796-802. [CrossRef]

6. Ainsworth, E.A.; Yendrek, C.R.; Skoneczka, J.A.; Long, S.P. Accelerating yield potential in soybean: Potential targets for biotechnological improvement. Plant Cell Environ. 2012, 35, 38-52. [CrossRef]

7. Buttery, B.R.; Buzzell, R.I. The relationship between chlorophyll content and rate of photosynthesis in soybeans. Can. J. Plant Sci. 1977, 57, 1-5. [CrossRef]

8. Dordas, C.; Sioulas, C. Safflower yield, chlorophyll content, photosynthesis, and water use efficiency response to nitrogen fertilization under rainfed conditions. Ind. Crops Prod. 2008, 27, 75-85. [CrossRef]

9. Kim, S.; Schlicke, H.; Van Ree, K.; Karvonen, K.; Subramaniam, A.; Richter, A.; Grimm, B.; Braam, J. Arabidopsis chlorophyll biosynthesis: An essential balance between the methylerythritol phosphate and tetrapyrrole pathways. Plant Cell 2013, 25, 4984-4993. [CrossRef]

10. Brzezowski, P.; Richter, A.S.; Grimm, B. Regulation and function of tetrapyrrole biosynthesis in plants and algae. Biochim. Biophys. Acta 2015, 1847, 968-985. [CrossRef]

11. Kobayashi, K.; Masuda, T. Transcriptional regulation of tetrapyrrole biosynthesis in Arabidopsis thaliana. Front. Plant Sci. 2016, 7, 1811. [CrossRef] [PubMed]

12. Hudson, D.; Guevara, D.; Yaish, M.W.; Hannam, C.; Long, N.; Clarke, J.D.; Bi, Y.M.; Rothstein, S.J. GNC and CGA1 modulate chlorophyll biosynthesis and glutamate synthase (GLU1/Fd-GOGAT) expression in Arabidopsis. PLoS ONE 2011, 6, e26765. [CrossRef] [PubMed]

13. Chiang, Y.H.; Zubo, Y.O.; Tapken, W.; Kim, H.J.; Lavanway, A.M.; Howard, L.; Pilon, M.; Kieber, J.J.; Schaller, G.E. Functional characterization of the GATA transcription factors GNC and CGA1 reveals their key role in chloroplast development, growth, and division in Arabidopsis. Plant Physiol. 2012, 160, 332-348. [CrossRef] [PubMed]

14. Bastakis, E.; Hedtke, B.; Klermund, C.; Grimm, B.; Schwechheimer, C. LLM-domain B-GATA transcription factors play multifaceted roles in controlling greening in Arabidopsis. Plant Cell 2018, 30, 582-599. [CrossRef]

15. Reyes, J.C.; Muro-Pastor, M.I.; Florencio, F.J. The GATA family of transcription factors in Arabidopsis and rice. Plant Physiol. 2004, 134, 1718-1732. [CrossRef] 
16. Behringer, C.; Schwechheimer, C. B-GATA transcription factors - insights into their structure, regulation, and role in plant development. Front. Plant Sci. 2015, 6, 90. [CrossRef]

17. An, Y.; Zhou, Y.; Han, X.; Shen, C.; Wang, S.; Liu, C.; Yin, W.; Xia, X. The GATA transcription factor GNC plays an important role in photosynthesis and growth in poplar. J. Exp. Bot. 2019, 71, 1969-1984. [CrossRef]

18. Bi, Y.M.; Zhang, Y.; Signorelli, T.; Zhao, R.; Zhu, T.; Rothstein, S. Genetic analysis of Arabidopsis GATA transcription factor gene family reveals a nitrate-inducible member important for chlorophyll synthesis and glucose sensitivity. Plant J. 2005, 44, 680-692. [CrossRef]

19. Zhang, Z.; Ren, C.; Zou, L.; Wang, Y.; Li, S.; Liang, Z. Characterization of the GATA gene family in Vitis vinifera: Genome-wide analysis, expression profiles, and involvement in light and phytohormone response. Genome 2018, 61, 713-723. [CrossRef]

20. Zhang, C.; Hou, Y.; Hao, Q.; Chen, H.; Chen, L.; Yuan, S.; Shan, Z.; Zhang, X.; Yang, Z.; Qiu, D.; et al. Genome-wide survey of the soybean GATA transcription factor gene family and expression analysis under low nitrogen stress. PLoS ONE 2015, 10, e0125174. [CrossRef]

21. Hao, Q.N.; Zhou, X.A.; Sha, A.H.; Wang, C.; Zhou, R.; Chen, S.L. Identification of genes associated with nitrogen-use efficiency by genome-wide transcriptional analysis of two soybean genotypes. BMC Genom. 2011, 12, 525. [CrossRef] [PubMed]

22. Buttery, B.R.; Buzzell, R.I. Soybean leaf nitrogen in relation to photosynthetic rate and yield. Can. J. Plant Sci. 1988, 68, 793-795. [CrossRef]

23. Lawlor, D.W.; Lemaire, G.; Gastal, F. Nitrogen, Plant Growth and Crop Yield. In Plant Nitrogen; Lea, P.J., Morot-Gaudry, J.-F., Eds.; Springe: Berlin/Heidelberg, Germany, 2001; pp. 343-367. [CrossRef]

24. Fritschi, F.B.; Ray, J.D. Soybean leaf nitrogen, chlorophyll content, and chlorophyll a/b ratio. Photosynthetica 2007, 45, 92-98. [CrossRef]

25. Hawkins, J.A.; Sawyer, J.E.; Barker, D.W.; Lundvall, J.P. Using relative chlorophyll meter values to determine nitrogen application rates for corn. Agron. J. 2007, 99, 1034-1040. [CrossRef]

26. Behringer, C.; Bastakis, E.; Ranftl, Q.L.; Mayer, K.F.; Schwechheimer, C. Functional diversification within the family of B-GATA transcription factors through the leucine-leucine-methionine domain. Plant Physiol. 2014, 166, 293-305. [CrossRef]

27. Missbach, S.; Weis, B.L.; Martin, R.; Simm, S.; Bohnsack, M.T.; Schleiff, E. 40 S ribosome biogenesis co-factors are essential for gametophyte and embryo development. PLoS ONE 2013, 8, e54084. [CrossRef]

28. Richter, R.; Behringer, C.; Muller, I.K.; Schwechheimer, C. The GATA-type transcription factors GNC and GNL/CGA1 repress gibberellin signaling downstream from DELLA proteins and PHYTOCHROMEINTERACTING FACTORS. Genes Dev. 2010, 24, 2093-2104. [CrossRef]

29. Mara, C.D.; Irish, V.F. Two GATA transcription factors are downstream effectors of floral homeotic gene action in Arabidopsis. Plant Physiol. 2008, 147, 707-718. [CrossRef]

30. Ranftl, Q.L.; Bastakis, E.; Klermund, C.; Schwechheimer, C. LLM-domain containing B-GATA factors control different aspects of cytokinin-regulated development in Arabidopsis thaliana. Plant Physiol. 2016, 170, 2295-2311. [CrossRef]

31. Hudson, D.; Guevara, D.R.; Hand, A.J.; Xu, Z.; Hao, L.; Chen, X.; Zhu, T.; Bi, Y.M.; Rothstein, S.J. Rice cytokinin GATA transcription Factor1 regulates chloroplast development and plant architecture. Plant Physiol. 2013, 162, 132-144. [CrossRef]

32. Richter, R.; Bastakis, E.; Schwechheimer, C. Cross-repressive interactions between SOC1 and the GATAs GNC and GNL/CGA1 in the control of greening, cold tolerance, and flowering time in Arabidopsis. Plant Physiol. 2013, 162, 1992-2004. [CrossRef] [PubMed]

33. Naito, T.; Kiba, T.; Koizumi, N.; Yamashino, T.; Mizuno, T. Characterization of a unique GATA family gene that responds to both light and cytokinin in Arabidopsis thaliana. Biosci. Biotechnol. Biochem. 2007, 71, 1557-1560. [CrossRef] [PubMed]

34. An, Y.; Han, X.; Tang, S.; Xia, X.; Yin, W. Poplar GATA transcription factor PdGNC is capable of regulating chloroplast ultrastructure, photosynthesis, and vegetative growth in Arabidopsis under varying nitrogen levels. Plant Cell Tissue Organ Cult. 2014, 119, 313-327. [CrossRef]

35. Richter, R.; Behringer, C.; Zourelidou, M.; Schwechheimer, C. Convergence of auxin and gibberellin signaling on the regulation of the GATA transcription factors GNC and GNL in Arabidopsis thaliana. Proc. Natl. Acad. Sci. USA 2013, 110, 13192-13197. [CrossRef] 
36. Liu, X.; Li, Y.; Zhong, S. Interplay between light and plant hormones in the control of arabidopsis seedling chlorophyll biosynthesis. Front. Plant Sci. 2017, 8, 1433. [CrossRef]

37. Springer, N.M. Isolation of plant DNA for PCR and genotyping using organic extraction and CTAB. Cold Spring Harb. Protoc. 2010, 2010, pdb-rot5515. [CrossRef]

38. Chen, S.; Songkumarn, P.; Liu, J.; Wang, G.L. A versatile zero background T-vector system for gene cloning and functional genomics. Plant Physiol. 2009, 150, 1111-1121. [CrossRef]

39. Zhang, X.; Henriques, R.; Lin, S.S.; Niu, Q.W.; Chua, N.H. Agrobacterium-mediated transformation of Arabidopsis thaliana using the floral dip method. Nat. Protoc. 2006, 1, 641-646. [CrossRef]

40. Lescot, M.; Dehais, P.; Thijs, G.; Marchal, K.; Moreau, Y.; Van de Peer, Y.; Rouze, P.; Rombauts, S. PlantCARE, a database of plant cis-acting regulatory elements and a portal to tools for in silico analysis of promoter sequences. Nucleic Acids Res. 2002, 30, 325-327. [CrossRef]

41. Schmittgen, T.D.; Livak, K.J. Analyzing real-time PCR data by the comparative CT method. Nat. Protoc. 2008, 3, 1101-1108. [CrossRef]

42. Zhang, C.; Liu, J.; Zhang, Y.; Cai, X.; Gong, P.; Zhang, J.; Wang, T.; Li, H.; Ye, Z. Overexpression of SIGMEs leads to ascorbate accumulation with enhanced oxidative stress, cold, and salt tolerance in tomato. Plant Cell Rep. 2011, 30, 389-398. [CrossRef] [PubMed]

43. Xin, Z.; Yuxia, W.; Mingui, Z.; Jianxin, H. Response of photosynthesis function of salt cress and Arabidopsis to $\mathrm{NaCl}$ salt stress. Chin. Bull. Bot. 2007, 24, 154-160.

44. Yoo, S.; Cho, Y.H.; Sheen, J. Arabidopsis mesophyll protoplasts: A versatile cell system for transient gene expression analysis. Nat. Protoc. 2007, 2, 1565-1572. [CrossRef] [PubMed]

(C) 2020 by the authors. Licensee MDPI, Basel, Switzerland. This article is an open access article distributed under the terms and conditions of the Creative Commons Attribution (CC BY) license (http://creativecommons.org/licenses/by/4.0/). 